\title{
Capability Assessment for Introducing Component Reuse.
}

\author{
Hugo Rehesaar \\ Griffith University \\ Brisbane, Queensland, Australia \\ +61414597171 \\ hugo.rehesaar@griffithuni.edu.au
}

\begin{abstract}
Despite initial technical barriers having been overcome, organizational wide component reuse has not enjoyed universal acceptance. Research has identified social and organizational factors as probable causes. This paper describes the Social Factors for Reuse Model (SFR Model), a predictive capability model based on Keidel's triadic model of the organization. It determines an organization's readiness for the introduction of Component Based Software Engineering (CBSE); describing the social and organizational conditions that should be met to maximize the chances of successful implementation. A sample application of the Model is described.
\end{abstract}

Keywords: Component reuse, reuse capability assessment, component based software engineering,

\section{Introduction}

The reuse of interchangeable components for software systems development was first publicly discussed in 1968 at the NATO Science Committee Conference on Software Engineering: Concepts and Techniques, at Garmisch, Germany [1]. Mcllroy argued that a "components industry could be immensely useful" and described the benefits of the reuse of components. Since then, Component Based Software Engineering (CBSE) has been shown to have benefits which include improvement of quality, faster development, and reduction in costs of development and maintenance [2].

Initially, technical factors prevented its successful implementation. Over time, the technical barriers have been all but overcome, and individuals have enthusiastically embraced the reuse of components. Yet, the organization-wide implementation of component reuse has yet to be adopted universally. [3],[4],[5],[6],[7],[8].

Research suggests that the reasons are related to socio-organizational barriers [4],[9],[10]. In order to better understand these, there is a need for a model that formalizes the socio-organizational factors influencing the successful implementation of component reuse. The model described in this paper, The Social Factors for Reuse Model, is inspired by Keidel's Triadic Model of organizations [11] and consists of factors that influence the organization-wide implementation of component reuse in the software engineering environment. It is intended that it will serve as a capability model for assessing an organization's readiness for the implementation of CBSE. By applying this Model, the organization can better decide what must be done to prepare 
itself for Reuse. The results of the assessment can be entered into an appropriate model to determine the economic and practical feasibility of making the required changes. Thus a Go/No Go decision can be made with greater confidence than otherwise would be possible.

For the purposes of this Model, component based software engineering (CBSE) is defined as the development of a system by the reuse, wholly or partially, of existing components. These components are not restricted to software artifacts or programs. They may include all products of the software development process ranging from requirement specifications and designs through to test data and implantation plans. Processes are also included, for example the elicitation of users' requirements and training of users. The following terms are held to be synonymous with CBSE: organization-wide component reuse, systematic (component) reuse, and component based software development [12]. Object Oriented Development and the use of Commercial off the Shelf (COTS) components are specific implementations of CBSE.

\section{Background}

\subsection{Benefits of CBSE}

The expected benefits of the implementation of organization-wide component reuse are well documented and tend to highlight savings in time and cost to develop [13],[14],[15],[16],[17]. IEEE1517-1999(Rev. 2004) [2] lists the following: increase in productivity; shorten software development time; move personnel, tools, and methods more easily from project to project; reduce software development and maintenance costs; produce higher quality software products; improve software product interoperability; provide a competitive advantage to an organization that practices reuse.

It is not suggested that an organization would enjoy all of these benefits, however any one of these would provide a sufficient reason to implement CBSE.

\subsection{Non-technical Obstacles to Implementation}

Initially, researchers and practitioners identified the technical obstacles to the successful adoption of CBSE, requiring enhancements to existing technology as well as the development and introduction of new technology.

Through the elimination of many of the technical issues in the 1980's and 1990's, component based development gained favour with developers and, in the early 1990's became of growing interest to researchers in the field of software engineering [18], [19],[20],[21],[22],[23]. Brown and Wallnau [24] offer two reasons for this, stating "several underlying technologies have matured that permit building components and assembling applications from sets of those components [and] the business and organizational context within which applications are developed, deployed and maintained has changed" in favour of component reuse. 
More recently, 2009, it has been suggested [25] that the organization may have cultures that are not conducive to systematic reuse and may lack the means to change its infrastructure to support the processes of systematic reuse without major disruption to its business. "Producing original software is sometimes more well-regarded than reusing existing software. Changing attitudes and associated non-reuse behaviors can be difficult. Policy changes and capital investments, which require senior management to be firmly committed to the achievement of systematic reuse, may be necessary." [25]

Indeed, the MIT Center for Information Systems Research [26] held a special session during its Summer Session, June 2010, and determined that companies do not reuse for reasons relating to behavior, politics and corporate culture.

Hence, the cause for the continuing lack of successful adoption of organizational wide reuse appears to lie most likely in the areas of organization and culture.

\subsection{Reuse Models}

Many models for reuse have been proposed, but not necessarily developed, for example [27] in 1993 and more recently [28] in 2007 and [29] in 2009, as well as many more in between. Several models have been developed as far back as 1992 [30] and as recently as 2010 [31]. However, all of these propose or provide for analyses of existing reuse programs and are not intended as predictors of implementation success.

In the late 80's and early 90's, reuse economics gained favour. A review of 17 reuse economic models [32] observed that all the models described and analyzed postimplementation reuse. It recommended that further research should be undertaken into models that would enable not just the evaluation of, but more importantly, the prediction of the costs, return-on-investments and other indicators. The Social Factors for Reuse Model is one such predictive model.

\section{The Social Factors for Reuse Model}

\subsection{Evolution of the SFR Model}

The SFR Model was inspired by the Triadic Model of the Organization by Robert Keidel [11]. The triadic shape "counterposes the three critical organizational variables: autonomy, control and co-operation" [11]. These correspond to the ways people interact.

1. Autonomy relates to working on your own.

2. Control relates to a hierarchical structure.

3. Co-operation relates to working together towards a common goal.

This structure is well established in management literature and practice [11].

The influence on the implementation of CBSE of the 36 factors that comprise the Model was obtained from literature and in-depth interviews with experienced software engineers. 
The Triadic Model is used to analyze the current state of an organization and also describe the ideal state it needs to be in to successfully implement CBSE, as described in 3.4. By comparing the two states, a determination can be made as to what must be changed in order for the organization to best prepare itself for CBSE implementation..

\subsection{Structure of the Model}

In the Social Factors for Reuse Model, the Organization is represented by a three tiered structure - see Table 1.

(b) Control

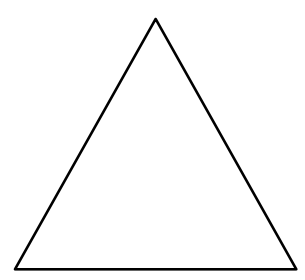

(a) Autonomy

(c) Co-operation

Figure 1: The Attribute Triad

Tier 1: The Organization is firstly broken down into 2 Dimensions.

(A) Organizational Strategy is concerned with planning and describes the proposed future state of the organization. (B) Organizational Implementation is concerned with processes and describes how the organization expects to achieve the future state.

Tier 2: Each of the Dimensions is further broken down into 6 Attributes: a total of 12. The Attributes for the SFR Model are based on the Keidel Model [11] and been adapted to align them with the domain of software engineering.

Tier 3: Each Attribute comprises of 3 Factors, for a total of 36 factors. The three factors in each Attribute correspond to

a) Autonomy,

b) Control, and

c) Co-operation.

The position of an organization on the triangle, Figure 1, describes its organizational behavior. To effectively manage an organization, a balance must be stuck among these three variables. For any given situation, the decision must be made as to which of these three is the most important. At best, two can share importance, but not all three. The triangular representation of the factors illustrates that you cannot fulfill all three factors at the same time.

An organization that can be described by one of the corners, is said to exhibit $100 \%$ of that factor. 
An organization that can be described by a point along one of the sides, will exhibit a combination of the two factors at the corners of that side.

The centre area represents an organization that exhibits equal amounts of each of the three factors. Porter describes such an organization as one that "engages in each generic strategy, but fails to achieve any of them”. [33]

\subsection{Attribute Co-relations.}

The Attributes are not independent of each other and therefore cannot be assessed independently. Table 1 shows the co-relations. They are

1) Meeting System, Decision System

2) Social Values, Organization's Expectations, Employee's Expectations, Reward System

3) Employee's Expectations, Developmental Plan

4) Reporting Lines, Physical Layout, Information Flow

\subsection{The 'Ideal' Candidate for Reuse Implementation.}

The SFR Model, summarized in Table 1, can be used to describe a generic ideal state for an organization that is about to embark on the implementation of organizational component reuse. The exact definition of 'ideal' is dependent on a number of factors. For any particular organization, this ideal state is called the 'goal state'.

The SFR Model is not all-inclusive. This is not a failure of the model, but rather it is intentional. No two organisations are alike. This applies to how they behave now and in the future. Each organization that embarks on change will do it from a different base and for different reasons. This was part of Keidel's original design for his model, and this philosophy has been adopted for the SFR Model. Some organizations have a need for extra attributes that are peculiar to their products, process, or environment, while others do not have the need for all of the attributes. An organization that enjoys a monopoly, for example an internal IT department and sole supplier to the organization as a whole, would not usually consider its Competitive Strategies. In like manner, if an organization does not have a mature training function, it may need to consider that as an additional attribute. One of the strong points of the SFR Model is that it allows for tailoring to the particular needs of the subject organization, resulting in an organization specific goal state. 
Table 1: The Social Factors for Reuse Model

(Breakdown of the Organization Showing the Influence of Factors.)

Attribute co-relations are shown thus: (99). Like numbers connect the attributes that are co-related.

\begin{tabular}{|c|c|c|}
\hline \multicolumn{3}{|c|}{ A. ORGANIZATIONAL STRATEGY DIMENSION } \\
\hline ATTRIBUTES & FACTORS & INFLUENCE \\
\hline \multirow{3}{*}{$\begin{array}{l}1 \text { Stakeholders (1) } \\
\text { For whose benefit does the } \\
\text { Organization exist? }\end{array}$} & 1a Customers/ End users & Zero \\
\hline & 1b Managers & positive \\
\hline & 1c Developers & positive \\
\hline \multirow{3}{*}{$\begin{array}{l}2 \text { Social Values ( } 2) \\
\text { What behavior does the organization } \\
\text { reward? }\end{array}$} & 2a Diversity of Approach & Negative \\
\hline & 2b Uniformity of Identity & Negative \\
\hline & 2c Complementarity & positive \\
\hline \multirow{3}{*}{$\begin{array}{l}3 \text { Organization's Expectations (2) } \\
\text { - from its employees. }\end{array}$} & 3a Self-reliance & Negative \\
\hline & 3b Compliance & positive \\
\hline & 3c Collaboration & positive \\
\hline \multirow{3}{*}{$\begin{array}{l}4 \text { Employee's Expectations (2) (3) } \\
\text { - from the organization. }\end{array}$} & 4a Opportunity & Negative \\
\hline & 4b Security & positive \\
\hline & 4c Community & positive \\
\hline \multirow{3}{*}{$\begin{array}{l}5 \text { Developmental Plan (3) } \\
\text { How does the organization grow? }\end{array}$} & 5a From Outside & Negative \\
\hline & 5b From Inside & positive \\
\hline & 5c In Partnership & positive \\
\hline \multirow{3}{*}{$\begin{array}{l}6 \text { Competitive Strategies } \\
\text { How does the organization compete? }\end{array}$} & 6a Differentiation & positive \\
\hline & 6b Cost & positive \\
\hline & 6c Flexibility & Negative \\
\hline \multicolumn{3}{|c|}{ B. ORGANIZATIONAL IMPLEMENTATION DIMENSION } \\
\hline \multirow{3}{*}{$\begin{array}{l}7 \text { Reporting Lines (4) } \\
\text { What is the form of our Reporting } \\
\text { and Communications relations? }\end{array}$} & 7a Flat/clear Lines & Zero \\
\hline & 7b Steep/clear Lines & Negative \\
\hline & 7c Flat/amorphous Lines & positive \\
\hline \multirow{3}{*}{$\begin{array}{l}8 \text { Physical Layout (4) } \\
\text { What interaction does our physical } \\
\text { design encourage? }\end{array}$} & 8a Independent Action & Negative \\
\hline & 8b Programmed Action & Negative \\
\hline & 8c Spontaneous Action & positive \\
\hline \multirow{3}{*}{$\begin{array}{l}\text { Information Flow (4) } \\
\text { How does our work/information } \\
\text { Flow? }\end{array}$} & 9a Pooled & Negative \\
\hline & 9b Sequential & Negative \\
\hline & 9c Reciprocal & positive \\
\hline \multirow{3}{*}{$\begin{array}{l}10 \text { Reward System (2) } \\
\text { What behaviors are reinforced } \\
\text { financially and non-financially? }\end{array}$} & 10a Individualistic & Negative \\
\hline & 10b Hierarchical & Zero \\
\hline & 10c Mutual & positive \\
\hline \multirow{3}{*}{$\begin{array}{l}11 \text { Meeting System (1) } \\
\text { For what reason do people get } \\
\text { together? }\end{array}$} & 11a Forum & Negative \\
\hline & 11b Decision-making & positive \\
\hline & 11c Team-building & positive \\
\hline \multirow{3}{*}{$\begin{array}{l}12 \text { Decision System (1) } \\
\text { How does the organization exercise } \\
\text { authority? }\end{array}$} & 12a Delegatory & positive \\
\hline & 12b Mandatory & Negative \\
\hline & 12c Collaboratory & positive \\
\hline
\end{tabular}


As a minimum, the following should be considered when developing an organization's goal state: the size of the organization and subject I.T. division, company ownership (government, listed, or private), the industry sector it is in, the effect of the market, the country of origin and location (culture and socio-economics), and single or multi location. Any one of these will affect the choice of factors, their influence and consequent mitigation strategies. The following is presented as a generic Ideal.

With respect to the Organizational Strategy Dimension, such an organization can be expected to have empowered its managers and developers with the ability to exert an influence on the manner in which the implementation is to take place. It should promote a culture of complementarity where all components are produced with the expectation of possible future reuse. Adherence to standards and procedures should be promoted, as too the acceptance that its employees are valuable and should be cared for. Promotion is best done from inside and partnerships with other organizations should only be done if that organization already has a culture of reuse. It would benefit from promoting it products' unique characteristics and exercising cost control.

With respect to the Organizational Implementation Dimension, the organization should promote formal and informal communications between all employees and provide a physical environment that promotes ad hoc communications, resulting in sharing of ideas and solutions. Its reward system should encourage co-operation amongst employees in achieving the common goal of reuse. Its meetings should not be simply talk-fests, but result in meaningful and practical solutions to problems as well as promoting the formation of like-minded teams to implement the solutions. Rather than mandating, the organization should have a delegatory style of decision making based on the consensus of all those involved in the implementation.

\section{Application of the SFR Model}

For a model to be more than just a theory, it must be shown to be of practical use. This section describes its application to an organization that has had a history of failed CBSE implementations.

\subsection{Steps in the Application of the Model}

The following are the steps in the application of the SFR Model to assess the capability of an organization and better prepare it for the implementation of component reuse. Figure 2 shows the steps as a flowchart.

1. Use the SFR Model to assess the initial organizational state prior to the consideration of CBSE implementation - $\mathrm{OS}_{\mathrm{I}}$.

2. Use the SFR Model to develop the model of the goal state of the organization $-\mathrm{OS}_{\mathrm{G}}$. This model represents the state in which the organization should be in order to maximize its likelihood of successful implementation.

3. Compare the results from Steps 1 and 2 to determine the difference between the initial and goal states. This is an assessment of the organization's initial 
capability to successfully implement a reuse program - OC . It will describe the changes required to increase the likelihood of success in implementing CBSE.

4. Implement the changes required to better prepare the organization for CBSE implementation, resulting in a prepared organization. As an option, an economic model can be applied prior to implementing the changes.

5. Use the SFR Model to assess the prepared organization's state - OS $_{\mathrm{p}}$.

6. Assess the prepared organization's capability by comparing the prepared state with the goal state, resulting in the Prepared Organization's Capability $\mathrm{OC}_{\mathrm{P}}$.

A major part of the predictive nature of the SFR Model is the feasibility assessment of the organizational change required to increase the likelihood of implementation success. There are many economic models for reuse, but they all relate to an organization that is already practicing reuse [32]. What is required is a model that is designed to be used pre-implementation. One such model that appears to be suitable is Benefit Cost Analysis [34]. The unique feature of this method is that it focuses on the benefits first and then the cost to achieve them. Jules Dupuit, an eighteenth century French economist, was the first to propose the concept central to benefit cost analysis of identifying the correct project benefits, and then the cost measurement criteria through demand and supply price mechanism. Applied to the introduction of reuse, it forces the quantification of the benefits of implementing reuse and so facilitates the decision to spend, or not to spend, the time and money needed for the required organizational change. A description of the Benefit Cost Model as it can be applied to IT projects is provided in [34].

\subsection{A Case Study}

This section presents a summary of an application of the SFR Model, describing the data collection method, results and recommendations for improving the subject organization's position with respect to the implementation of organization wide component reuse.

The data collection methods are based on those suggested by Keidel [11] and are presented as examples only. Both the date collection methods and the recommendations will vary for each individual application of the SFR Model. Other methods may prove to be more successful, depending on the subject organization. The recommendations also may vary depending again on the subject organization and of course on the particular results obtained.

The subject organization is a large multi-national company, which develops embedded software for companies world-wide in a number of industries, as well for its own products. The implementation of CBSE has had mixed results, ranging from successes to outright failures. The successes have not been as a result of well thought out plans. 


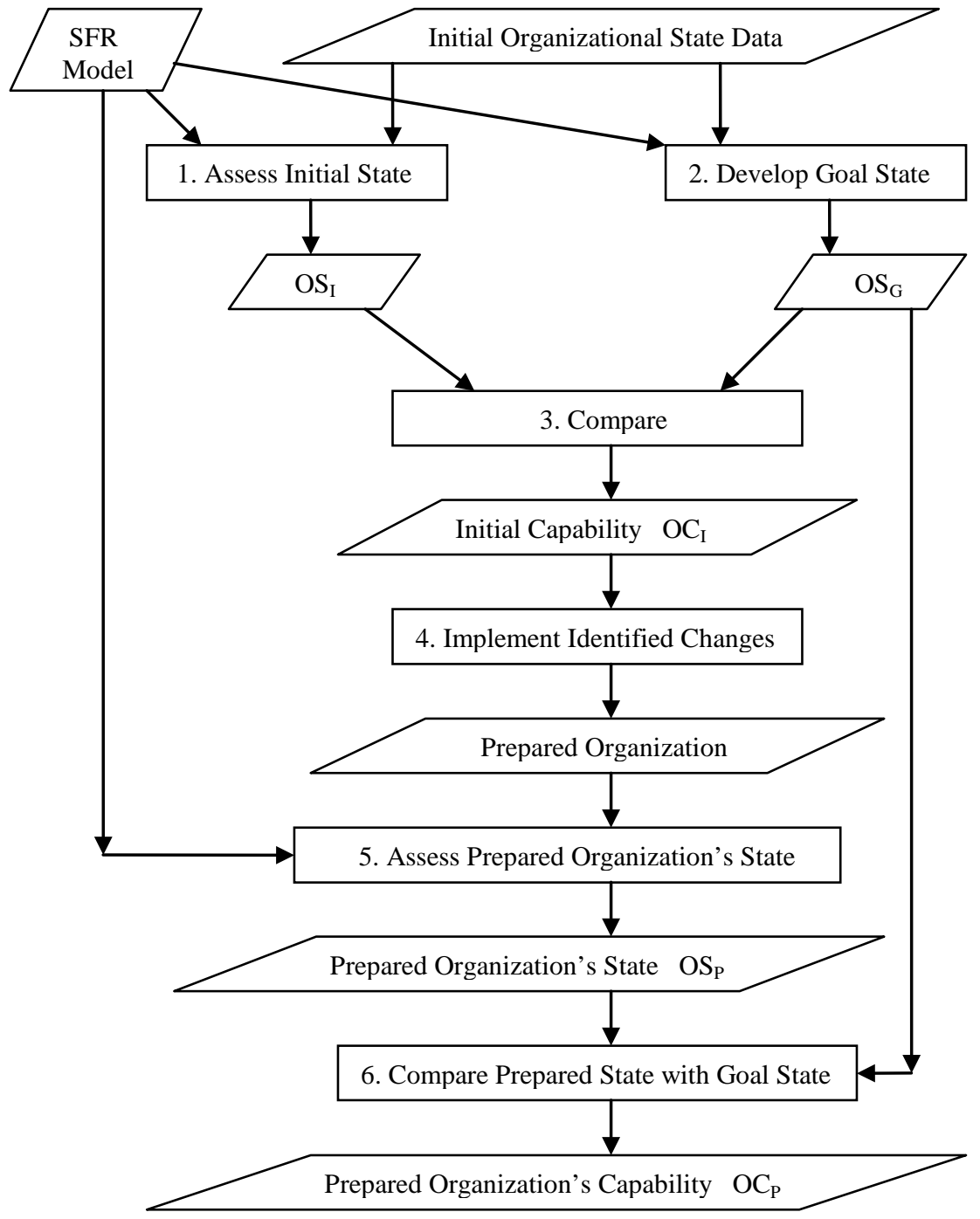

Figure 2: Flowchart of the Application of the SFR Model.

The subject division, since its failed attempt, has implemented a number of changes, which it believes will improve its implementation capability - OC. The following is a summary report of the subsequent application of the SFR Model to this division to assess its new state. The assessment commenced at Step 5 and used the generic ideal state as the goal state. 


\section{Organizational Strategy Dimension}

\subsubsection{Attribute 1: Stakeholders.}

Data Collection Method: Analysis of various archival documents, providing information on the relative actual importance of the various stakeholders. In-depth interviews with staff, both managers and developers.

Results: Documentation stated that staff at all levels have been given the necessary delegation and authority appropriate to their jobs. This however was not supported by many of the managers and developers, who reported that their managers often mandated actions that should have been the decision of the sub-ordinate. They felt that they did not have the influence that they were meant, and consider that they deserve, to have.

Recommendations: It is necessary for the organization to realize that there is difference between statement and action. Their current attitude has resulted in dissatisfaction among employees and is seen as one of the reasons for the previous failure. A review of the Decision System is recommended. Refer to Attribute 12: Decision System.

\subsubsection{Attribute 2: Social Values.}

Data Collection Method: In-depth interviews with managers representing all levels. Results: There was unanimous agreement at (almost) all levels that working together was the key to success both for the individual and the company. There was disagreement amongst those in the highest of management, with some supporting cooperation. A small majority felt that the company could not advance if it did not have some individual 'high flyers'. These people were felt to ward off risks of complacency.

Recommendations: It is felt that there is no threat to CBSE implementation and so there is no recommendation to change.

\subsubsection{Attribute 3: Organization's Expectations.}

Data Collection Method: In-depth interviews with managers representing all levels.

Results: Apart high flyers (described in Attribute 2: Social Values), the management expressed an expectation of compliance and promoted an environment of collaboration.

Recommendations: Organization Expectations are satisfactory and no recommendations are offered.

\subsubsection{Attribute 4: Employees' Expectations.}

Data Collection Method: In-depth interviews with managers and developers.

Results: All but a few (and these corresponded with the individual's identified by management as being 'high flyers' - see Attribute 2: Social Values) were in alignment, to varying degrees, to the factors of 'security' and 'community'.

Recommendations: Employee Expectations are satisfactory and no recommendations are offered. 


\subsubsection{Attribute 5: Developmental Plan.}

Data Collection Method: Analysis of archival documents, providing historical information on what plans, events and reasons for recruitment and partnerships/mergers. In-depth interviews with staff.

Results: Staff at all levels admitted that promotion did occur from within. This was confirmed by the documents. However, they were resentful when an outsider was brought in at a managerial level and was not aware of the existing culture (social values). Management stated that this was sometimes done in order "to change the way things were done. To shake things up". They were not concerned that some staff did not like it as they were often the reason for the required change. The company very rarely participated in mergers or takeovers, preferring to start a new division from within.

Recommendations: Generally, the plan is satisfactory. However, care must be taken that the engineered change is necessary as a whole and not simply to facilitate the removal of undesired personnel. There are less disruptive ways to do this.

\subsubsection{Attribute 6: Competitive Strategies.}

Data Collection Method: Analysis of archival documents. In-depth interviews with management. Discussions with customers/clients to provide the 'other' view, that is, the success or not of these strategies.

Results: The division had an established culture of producing 'differentiated and quality' products. This was supported by the majority of the customers/clients. All staff recognized that a cheaper product was beneficial and were willing to do what was needed to achieve this, without sacrificing quality.

Recommendations: Organization Strategies are satisfactory and no recommendations are offered.

\section{Organizational Implementation Dimension.}

\subsubsection{Attribute 7: Reporting Lines.}

Data Collection Method: Analysis of the organizational chart and in-depth interviews with manager and developers.

Results: Although the company as a whole exhibits all three styles of Reporting Lines, this division has flat/amorphous reporting. The formation of individual teams is flexible, dependent on specific project needs and availability of appropriates experienced staff.

Recommendations: Reporting Lines are satisfactory and no recommendations are offered.

\subsubsection{Attribute 8: Physical Layout.}

Data Collection Method: Determination of who should be talking to whom by reference to project records and organizational chart. Comparison with locations of these personnel.

Results: As this is a multi-national company, it was found that many projects spanned countries and time zones. Small groups of developers were co-located (and there was evidence of successful reuse within these groups), but many who would be expected to have face-to-face communication were in fact in different time zones and countries. 
Staff said that this did not present a problem because of excellent electronic communications being in place. Some found that the use of such facilities was preferred, because it allowed for the other party to respond after thought had been given to the problem. A small number found that these did not fully replace face-toface.

Recommendations: While the physical layout is not ideal, communications seem to be as good as they can be given the international nature of the company and its customers. No recommendations are offered.

\subsubsection{Attribute 9: Information Flow.}

Data Collection Method: Network analysis of staff working on similar projects to determine actual flow to compare with expected communication patterns based on the needs of the projects.

Results: The expected patterns of communication co-related well with the actual information flows.

Recommendations: Information Flow is satisfactory and no recommendations are offered.

\subsubsection{Attribute 10: Reward System.}

Data Collection Method: In-depth interviews with staff (managers and developers) and analysis of archival documents relative to pay scales and bonuses over the previous three years.

Results: Bonus schemes do not exist in this division and employee's are accepting of this. Pays are reviewed annually and set commensurate with the employee's job description and their past performance. Employee's are satisfied with this.

Recommendations: Management could consider a rewards system for reuse implementation, but considering that employee's appear satisfied with current arrangements, this is a suggestion only and no recommendations are offered.

\subsubsection{Attribute 11: Meeting System.}

Data Collection Method: Ratios of the number of meetings and total time (manhours) spent on each type of meeting over the previous month. In-depth interviews with staff, both managers and developers.

Results: While the mangers saw the meetings as positive decision-making and teambuilding in nature (an opinion supported by documentation), this was not the opinion of the developers and lower managers. The decisions from the meetings were often subsequently over-ruled by the manager, for reasons that were seen by the manager as practical, and by the sub-ordinates as taking away their influence. They were in agreement, that despite the claimed best intentions of the management, the reality was that the majority of meetings ultimately resulted in being only "talk-fests" or forums. They also believed that the managers did not have the authority to over-ride meetings' decisions, but felt powerless to act.

Recommendations: The disparity between the apparent meeting style and the implementation is a real concern. If it is not possible to stop managers over-riding meeting decisions, then the façade of co-operation should be discontinued. Meetings should be openly announced as forums and managers authority made clear. This is not a good solution. Although it clarifies an anomalous situation for the staff, it promotes 
a mandatory style of management, which usually has a negative influence. Enforcing the finality of decision made at meetings is the better alternative, though in practice more difficult to enforce.

\subsubsection{Attribute 12: Decision System.}

Data Collection Method: Analysis of archival documents over the previous three months (meeting records, authorization and delegation lists, project plans and memos) to determine the decisions made, how they were made and by whom. In-depth interviews with staff.

Results: The majority of decisions were made collaboratorially. However, there was a tendency to mandate major decisions, including that of implementing reuse. Delegation, although practiced widely, also tended to use a mandatory style and often the delegation was not welcomed by the recipient, often being interpreted as a test of ability and in a few instances as 'traps' to facilitate the dismissal of the employee. It was found that managers often over-rode decisions made by a team and their immediate subordinates.

Recommendations: At the very least, the next decision to implement reuse should involve all divisional personnel. The details of the implementation must be agreed to in a collaboratory way. Delegation should be seen as a reward and have the agreement of the recipient. Refer to Attribute1: Stakeholders.

\section{Concluding Remarks}

The Division seems to have improved its Implementation Capability, although the extent of this could not be determined with any precision owing to a lack of reliable records of the changes that had already been made. There remain a number of areas that require attention, which relate to the Attributes of Stakeholders, Development Plan, Meeting System and Decision System. These centre on a disparity between the organization's stated intentions and the implementation of them, leaving staff "on edge" and with a decreasing openness to change. It is recommended that an assessment be done to determine the economic and social/organizational viability of implementing these changes prior to the next attempt to implement division wide component reuse.

\section{Further Development}

As it should be with any such model, the SFR Model is under regular review and a number of developments and enhancements are described here.

The current Model also considers only the influence that the presence of a Factor exerts on the implementation of CBSE. While it can be generalized that the absence of a Factor equates to the absence of an influence, this is not always the case. A Factor can also exert an influence by its absence, for example the absence of a Development Plan (Attribute 5) can have a negative influence on the Employee's Expectations 
(Attribute 4). Work is continuing to determine the influence of the absence of the Attributes and Factors.

A similar situation arises when, for example, the result of a Collaboratory Decision (Factor 12c) is to not implement component reuse, even though management is in favour of it. While normally the Collaboratory Decision System would exert a positive influence, in this case the influence would be negative. Such instances need to be incorporated into the Model.

As the SFR Model is a predictive model, it lends itself to being coupled with an appropriate costing model for the changes that are identified as necessary for successful implementation. The Benefit Cost Model [46] appears to be well suited to this task. Further work is required to determine whether this or another cost model is most suitable.

\section{Conclusions}

Systematic component reuse has yet to be successfully adopted by the vast majority of organizations, even though individuals have embraced it enthusiastically for decades. The initial technical barriers have been all but overcome and the current barriers appear to be based on socio-organizational factors.

The Social Factors for Reuse Model was developed from a perceived need for a model for Component Based Software Engineering implementation that considered the social and organizational factors. The SFR Model comprises 36 factors that provide a structure to describe an ideal state for an organization, against which it can be assessed to determine its capability to embark on the implementation of CBSE. Such an ideal state describes an organization that is not only sympathetic to the concept of reuse, but also actively promotes its organization wide implementation.

When coupled with a suitable costing model, the organization can make an informed decision whether or not to proceed with CBSE implementation. This then has the potential to greatly reduce the number and cost of unsuccessful CBSE implementations.

\section{References}

[1] McIlroy, MD. Mass-produced software components. In J.M. Buxton, P. Naur and B. Randell, (eds) Software Engineering Concepts and Techniques, 1968 Nato Conference on Software Engineering, (Garmisch, Germany, 1968), 1976, 88-98.

[2] IEEE1517-1999(Rev. 2004). IEEE1517 Standard for Information Technology Software Life Cycle Processes - Reuse Processes: 1999, reaffirmed 2004. Software Engineering Standards Committee of the IEEE Computer Society, USA. 2004.

[3] Garcia, V.C., Lucrédio, D., Alvaro, A., de Almeida, E.S., de Mattos Fortes, R.P. and de Lemos Meira, S.R. Towards a maturity model for a reuse incremental 
adoption. In The $1^{\text {st }}$ Brazilian Symposium on Software Components, Architecture and Reuse. (Campinas, Sâo Paulo, Brazil, 2007), 61-74.

[4] Chroust, G. Motivation in component-based software development. In C. Ghaoui (ed) Encyclopedia of Human Computer Interaction. Idea Group Reference, Hershey, PA and London, 2006.

[5] Sherif, K. and Vinze, A.S. Barriers to adoption of software reuse: a qualitative study. Information and Management, 41, 2, (2003), 159-175.

[6] Ravichandran, T. (1999), Software reuseability as synchronous innovation: a test of four theoretical models. European Journal of Information Systems. 8, 1999, 83-199.

[7] Allen, P. Using CBD to improve your business. In Component Strategies, 2, 1, SIGS Publications, New York, July 1999.

[8] Kim, Y. and Stohr, E.A. Software reuse: Survey and Research Directions. Princeton, NJ, USA, Princeton University Press, 1998.

[9] Crooks, M. Capitalizing on component reuse. Component Strategies, July, 1999. New York. ISSN: 10993673, 44.

[10] Kunda D. and Brooks L. Human, social and organizational influences on component-based software engineering. In Proceedings of the $21^{\text {st }}$ International Conference on Software Engineering. (Los Angeles, May, 1999). IEEE Computer Society.

[11] Keidel, R. Seeing Organizational Patterns. $2^{\text {nd }}$ edition, Beard Books, Washington, USA, 2005.

[12] Haines, C.G., Carney, D., and Foreman, J. Component-based Software Development/COTS Integration, Software Engineering Institute, Carnegie Mellon University, 1997.

[13] Waguespack, L. and Schiano, W.T. Component-based IS architecture. Information Systems Management. 21, 3, (2004), 53-60.

[14] Vitharana, P. Risks and challenges of component-based software development. Communications of the ACM. 46, 8, (2003)67-72.

[15] Dué, R.T. The economics of Component-based Development. Information Systems Management. 17, 1, 92-95.

[16] Kunda, D. and Brooks, L. Assessing organizational obstacles to componentbased development: a case study approach. Information and Software Technology. 42, 10, 2000, 715-725.

[17] Pour, G. Moving toward component-based software development approach. In Proceedings of the 27th International Conference on Technology of ObjectOriented Languages and Systems, (Beijing, China, September 1998), IEEE Computer Society Press, Los Alamitos, California, USA, 296-300.

[18] Hall, P.A.V. Architecture driven software reuse, Information and Software Technology, 41, 1999.

[19] Kiely, D. Are components the future of software? IEEE Computer, 31, 2, 1998, 10-11.

[20] Szyperski, C. Component Software: Beyond Object-oriented Programming. Addison Wesley Longman, Reading, Mass. USA, 1998.

[21] Sametinger, J. Software Engineering with Reusable Components. Springer Verlag, 1997. 
[22] Aoyama, M. Componentware: building applications with software components, Journal of the International Process Society of Japan, 37, 1, 1996, 71-79.

[23] Brown, A.W. Component Based Software Engineering IEEE Computer Press, 1996.

[24] Brown, A.W., and Wallnau, K.C. The current state of CBSE. IEEE Software, 15, 5, (Sept/Oct 1998), 37-46.

[25] IEEE1517-2009 D2. IEEE1517 Standard for Information Technology - Software Life Cycle Processes - Reuse Processes: 2009 D2. Software Engineering Standards Committee of the IEEE Computer Society, USA. 2009

[26] MIT, 2010 Summer Session, Barriers to design, process and code reuse. MIT Center for Information Systems Research, June, 2010. Downloaded $12^{\text {th }}$ July, 2010. http://www.ciodashboard.com/it-strategy/13-barriers-to-reuse/

[27] Davis, T. The reuse capability model: a basis for improving an organization's reuse capability. In The Proceedings of Advances in Software Reuse. (Lucca, Italy, March 24-26, 1993) IEEE.

[28] Alvaro, A., de Almeida, E.S. and Meira, S.L. A software component maturity model. In Proceedings of the $33^{\text {rd }}$ EUROMICRO Conference on Software Engineering and Advanced Applications, (Lübeck, Germany, Aug 27-31, 2007), SEAA 2007, IEEE, 2007, 83-90.

[29] Tripathi, A.K. and Ratneshwer. Some observations on a maturity model for CBSE. In 14th IEEE International Conference on Engineering Complex Computer Systems, (Potsdam, Germany, June 02-04, 2009), ICECCS2009, IEEE, New York, USA, 274-282.

[30] Creps, R.E, Simos, M.A and Prieo-Diaz, R. The STARS conceptual framework for reuse processes. In The Proceedings of the Fifth Annual Workshop on Software Reuse. (Palo Alto, California, USA, October, 1992) WISR'92.

[31] K.S., Jasmine and Vasantha, R. A new capability maturity model for reuse based software development process. IACSIT International Journal of Engineering and Technology, 2, 1, (February, 2010), ISSN 1793-8236, 112-116.

[32] Lim, W.C. Reuse economics: a comparison of seventeen models and directions for future research. In Proceedings of the $4^{\text {th }}$ International Conference on Software Reuse. (1996), IEEE Computer Society, DC, USA, 41-50.

[33] Porter, M. Competitive Advantage: Creating and Sustaining Superior Performance. Free Press, New York, 1985, 16.

[34] Rehesaar, H. and Mead, A. An extension of Benefit Cost Analysis to IT Investments. Business Review, Cambridge, Vol. 4, No. 1, Summer, 2005, Cambridge, Ma., USA. ISSN 1553-5827, 89-93. 